http://doi.org/10.15359/ree.14-2.7

\title{
La analogía como estrategia cognitiva que favorece la comprensión lectora en textos expositivos
}

\section{The Analogy as a Cognitive Strategy that Encourages Reading Comprehension in Expository Texts}

\author{
José Viveros Márquez ${ }^{1}$ \\ Escuela Primaria el Niño Azteca \\ México \\ vive1903jose@hotmail.com
}

Recibido 15 de abril de 2010 • Aceptado 24 de junio de 2010

\begin{abstract}
Resumen. La presente investigación surge a partir de la situación actual de la lectura en México, dado que la realidad contextual a nivel local y nacional se ha tornado crítica respecto a este tema. Los resultados en pruebas estandarizadas como ENLACE (Evaluación Nacional del Logro Académico en Centros Escolares) y PISA (Programa Internacional para la Evaluación de los Estudiantes) han demostrado que los estudiantes mexicanos del nivel básico presentan problemas de comprensión de textos. Ante esta realidad se hace un estudio descriptivo-cualitativo sobre el uso de analogías en la lectura como estrategia para la comprensión lectora, mediante la aplicación de una encuesta etnográfica, un cuestionario y un Modelo Pedagógico para el uso de Analogías en la lectura. El estudio retoma los postulados teóricos del Constructivismo, en referencia a Piaget (1969), Vigotsky (1997) y Ausubel (2002). Los resultados encontrados reflejan que el uso de analogías favorece la comprensión lectora de los alumnos de cuarto grado, a partir de un texto expositivo que compara la evolución del hombre y la metamorfosis de una mariposa.
\end{abstract}

Palabras clave. Analogías, comprensión lectora, estrategias de lectura, constructivismo.

Abstract. This research arises from the current state of reading in Mexico, because the contextual situation to local and national level has become critical on this issue. Results on standardized tests such as ENLACE (National Assessment of Academic Achievement in Schools) and PISA (Program for International Student Assessment) have shown that Mexican basic level students have text comprehension problems. Given this reality, this is a qualitative descriptive study on the use of analogies in reading as a strategy for reading comprehension, through application of an ethnographic survey, a questionnaire and the application of a pedagogical model for the use of analogies in reading. The study incorporates the theoretical tenets of Constructivism, referring to Piaget (1969), Vygotsky (1997) and Ausubel (2002). The results show that the use of analogies promotes reading comprehension in 4th grade students, from an expository text that compares the evolution of man and the metamorphosis of a butterfly.

Key words. Analogies, reading comprehension, reading strategies, constructivism.

Licenciado en Educación Primaria por la Escuela Normal Rural "Lázaro Cárdenas del Río". Profesor de educación primaria con experiencia en educación rural. Maestro en Ciencias de la Educación por la Universidad del Valle de México. Asesor pedagógico del Centro de Promoción Humana "Talita Kum". Consultoría y Asesoría Psicopedagógica CAPS. Actualmente doctorante en Ciencias Sociales en el Instituto de Ciencias Sociales y Humanidades (ICSHu), de la Universidad Autónoma del Estado de Hidalgo. 


\section{Introducción}

La relevancia que ha tenido la lectura dentro de los programas educativos en México ha motivado la reformulación y el diseño de planes y programas de estudio, de libros de texto, capacitación del profesorado, entre otros aspectos; además de su implicación en la praxis educativa, pues es necesario que dentro de las escuelas primarias se busquen las formas más adecuadas para lograr que los alumnos adquieran, afiancen y desarrollen un buen nivel de lectura, no solo en los aspectos formales -fluidez, dicción, entonación, etc.-, sino también, en la estructura profunda de esta actividad -lo que ocurre cuando alguien comprende un texto- y cómo llega a hacerlo.

Hoy por hoy, conocemos el proceso de la lectura y todo lo que implica. Psicólogos y especialistas en educación han teorizado o explicado lo que sucede cuando una persona lee y cómo llega a la comprensión; sin embargo, dentro de nuestro contexto social y educativo nos encontramos con alumnos que leen, pero no consiguen una comprensión total de lo leído. Tal como se ha constatado con las evaluaciones de PISA (Programa Internacional para la Evaluación de los Estudiantes) que se aplican en México desde el año 2000, en cuyos resultados nuestro país ha ocupado los últimos lugares en habilidades de lectura.

En México se han implementado diversos programas de lectura y se han diseñado estrategias para superar las deficiencias en lectura y escritura. A partir de 1970, mediante programas como PALEM (Propuesta para el Aprendizaje de la Lengua Escrita y la Matemática), PRONALES (Programa Nacional para el Aprendizaje de la Lectura y la Escritura) y, más recientemente, el PNL (Programa Nacional de Lectura), esta competencia toma otro sentido y se constituye en una construcción de significados. Posteriormente, y a partir del Acuerdo Nacional Para la Modernización Educativa de 1989-1993, la lectura da un giro muy significativo: se deja a un lado el enfoque formativo y se pasa a un enfoque comunicativo y funcional, con énfasis en la comprensión lectora y la formación de hábitos de lectura. De esta manera se crean las bibliotecas de aula y los libros del rincón, y se editan libros para cada ciclo escolar.

En esta investigación se enfatiza que la comprensión lectora depende de la información no visual, esto es, de los conocimientos presentes en la estructura cognitiva, de acuerdo con el autor Smith (1989), postura paralela que se observa en la Teoría del Aprendizaje Verbal Significativo de Ausubel (2002).

El constructivismo es una de las teorías que explica el proceso de aprendizaje en los seres humanos. Se retoman así las aportaciones de autores como Piaget (1969), quien explica la línea natural de desarrollo, puntualizando los estadios de desarrollo cognitivo. Autores como Vigostky (1997) y Ausubel (2002) explican también el proceso de aprendizaje, se hace referencia a la línea cultural y al aprendizaje significativo.

La investigación realizada buscó la aplicación de la analogía como medio para la promoción de la comprensión lectora. Considerando el contexto social y cultural de la comunidad rural en la cual se desarrollaría la experiencia, así como la variedad de flora y fauna que la caracteriza, se recurrió al proceso de metamorfosis de las mariposas como elemento analógico. Debido a que los 27 alumnos de cuarto grado participantes conocen el proceso o saben de las diferentes etapas de cambio de las mariposas, o lo observan en el contexto, se determinó su utilización como analogía, para compararla con la evolución del ser humano. Se buscaba conocer el proceso seguido por los participantes cuando leen, así como las estrategias que toman en cuenta y determinar si su pensamiento es analógico o inferencial.

Los resultados encontrados en la fase del diagnóstico indican que la mayoría de los alumnos/as se muestran como lectores activos, pues siguen estrategias que los ayudan a la comprensión lectora. 
Asimismo muestran que tienen un pensamiento analógico, pues logran comparar información de campos de conocimientos similares, deducen nueva información y logran obtener inferencias correctas. Las inferencias permitieron que los participantes y las participantes obtuvieran conclusiones generales y globales respecto de un texto, a partir de la extracción de la idea más importante o central de un párrafo.

Con estos resultados se partió de un supuesto importante, el cual es que los niños y niñas de 10 años logran comparar y transferir información de un campo relacional conocido a otro en cierta medida similar, pero menos conocido. Esta premisa básica fundamentó la aplicación de la siguiente fase de la investigación, la cual consistió en el diseño del modelo pedagógico. Este retomó un texto expositivo referido a la evolución del ser humano, el cual se comparó con la metamorfosis de una mariposa. Ambos textos presentan semejanzas que permitieron analizarlos de manera paralela: se llevó a cabo el proceso de comparación entre ambos campos de conocimiento, tomando como campo base la metamorfosis de una mariposa y como campo objetivo la evolución del ser humano. Los resultados obtenidos permiten inferir que la analogía aplicada permitió a los alumnos mejorar, en gran medida, la comprensión del tema.

\section{Panorama general de la lectura en México}

En un estudio realizado por la UNESCO, México ocupa el penúltimo lugar en hábitos de lectura, con un promedio de 2.8 libros a/h, cifra muy alejada de los 25 volúmenes recomendados por este organismo internacional y del promedio de lectura de la sociedad japonesa, noruega, finlandesa y canadiense, que ocupan los primeros lugares a nivel mundial con 47 títulos per cápita (Gutiérrez, 2005, pp. 94-95).

Así mismo el Instituto Nacional de Evaluación Educativa indica que el 66,6\% de estudiantes de sexto grado de educación primaria presentan niveles bajos de lectura y solo el $12 \%$ de ellos, cuenta con las competencias lectoras para cubrir expectativas (INEE, 2006). Los resultados de PISA 2006 presentados por el INEE, (2007) sitúan a México en los últimos lugares de 57 países que fueron evaluados en habilidades de lectura y matemáticas, considerando que los alumnos mexicanos apenas y cuentan con las herramientas básicas para la comprensión de textos simples, extraen información literal y lógica, presentando problemas graves al enfrentarse a situaciones de lectura más complejas.

En esta evaluación de PISA 2006, en lectura, México ocupó el lugar 43 de 57 que participaron, en matemáticas lugar 48 y en ciencias ocupó el lugar 49. Cabe señalar que esta evaluación se ha aplicado en nuestro país en tres ocasiones, la primera en 2000, luego en 2003 y esta última en 2006. En lectura, el 21\% de los estudiantes se ubicaron en el nivel 0 (menos de 334.75 puntos), el 6\% alcanzó los niveles 4-5 y ningún alumno alcanzo el nivel 6. "México logra el nivel 2 en la escala global de lectura, lo que significa que los estudiantes son capaces de realizar tareas básicas de lectura, como localizar informaciones sencillas, realizar deducciones simples de distintos tipos, averiguar lo que significa una parte claramente definida de un texto y usar ciertos conocimientos externos para comprenderlo”. (Instituto Nacional para la Evaluación de la Educación en México [INEE], 2007, pp. 99-100).

Las estadísticas señaladas sólo indican que la falta de comprensión lectora es hoy un problema real generalizado en todo el país, en todos los contextos y en todos los niveles educativos. La sociedad reclama mejores resultados, pues en las evaluaciones aplicadas, hechas por organismos 
nacionales como ENLACE (Evaluación Nacional del Logro Académico en los Centros Escolares) e internacionales como PISA, se demuestra una deficiencia en el dominio de contenidos que se fundamentan en esta competencia, en la reflexión y el análisis de textos.

Un ejemplo concreto de la falta de comprensión lectora son los resultados de la prueba ENLACE y otras pruebas objetivas que se aplican a los alumnos, según las cuales la mayoría de los mismos presenta niveles muy bajos de lectura, ya que un porcentaje muy alto de los alumnos mexicanos obtienen resultados insuficientes y elementales, lo que indica que no adquirieron los conocimientos de la asignatura evaluada. Entre 2008 y 2009, en México, los alumnos con un nivel insuficiente y elemental (estudiantes que tienen un mínimo o no adquirieron los conocimientos de la asignatura evaluada) pasaron de 71.5 por ciento a 69,2 ; y aquellos con un nivel bueno o excelente (estudiantes que tienen dominio de los contenidos de la asignatura evaluada) aumentaron de 28,5 a 30,3 .

Las preguntas obligadas al respecto serían: ¿a qué se deben tales resultados?, ¿realmente estamos enseñando estrategias que ayuden los estudiantes a comprender los textos?, o ¿de qué depende que un alumno pueda rescatar el significado de más relevancia en una prueba estandarizada?, ¿por qué no es posible que encuentre relación entre la información propia y la del texto y de esta forma obtener inferencias correctas? etc.

Como se observa en los datos anteriores, la lectura es un problema real en nuestro país, por lo que es necesario replantear los medios y las estrategias que se han venido empleando para contrarrestarlo. Los programas de lectura hoy deben ser reorientados con énfasis en el desarrollo de la lectura de comprensión. No podemos soslayar la importancia cualitativa que debe desempeñar que los alumnos sean lectores competentes para sobresalir en su desempeño escolar: los alumnos deben comprender, no solo descifrar. Hace falta que mejoren en esta habilidad, ya que la deficiente comprensión lectora constituye uno de los factores que limita el desempeño de los alumnos en el trabajo continuo y en las pruebas de valoración.

\section{2. ¿Qué se entiende por comprensión lectora?}

La comprensión es una actividad propia de los seres humanos que consiste en la construcción de significados a partir de otros conocimientos. Comprender un texto va más allá de extraer y memorizar su información literal. Para comprender es necesario interactuar con la información y utilizar esa información con fines específicos. En esta interacción son importantes los conocimientos previos del lector. Sarmiento (1995) define a la comprensión como:

Un proceso constructivo, en el que la información de un estímulo o evento se empareja con otra información existente en la memoria del sujeto. A la información existente en la memoria del sujeto se le llama conocimiento del mundo y es este el que permite al lector inferir información que no está presente explícitamente en el texto, o en otras palabras, leer cosas que no están escritas, pero que formaban parte del mensaje del autor. (p. 22)

Para Smith (1989), la comprensión, tal y como se le concibe actualmente, es un proceso a través del cual el lector elabora un significado en su interacción con el texto. La comprensión a la que el lector arriba durante la lectura se deriva de sus experiencias acumuladas, experiencias que entran en juego y se ven modificadas a medida que decodifica las palabras, frases, párrafos e ideas del autor. 
La interacción entre el lector y el texto es el fundamento de la comprensión. En este proceso de comprender, el lector relaciona la información que el autor presenta con la información almacenada en su mente; este es el proceso de relacionar la información nueva con la antigua, es, en una palabra, el proceso de la comprensión.

Concuerdan autores (Smith, 1989; Ausubel, 2002) en que la comprensión es el proceso de elaborar el significado por la vía de aprender las ideas relevantes de un texto. Sin importar la longitud o brevedad del párrafo, el proceso se da siempre de la misma forma: mediante la identificación de las relaciones e ideas que el autor presente, entendiendo lo que se está leyendo, relacionando las nuevas ideas con ideas ya almacenadas en la memoria. Estos autores hacen énfasis en la información no visual y en los conocimientos previos, como elementos fundamentales para que se llegue a comprender un texto.

\section{Analogías y comprensión lectora}

Las analogías se han empleado como recurso para la enseñanza, tanto en la lectura como en la solución de problemas matemáticos. Las analogías, dentro de la lectura, se han usado como medio de comparación entre dos campos de conocimientos. Partiendo de esta premisa, se puede comparar y explicar el significado, función y cuidados del Sistema Inmunológico (S. I) quizá con un "soldado" que resguarda y protege a un Estado o Nación. Si analizamos las características o funciones del S. I. con las funciones de un soldado, podemos constatar que algo abstracto (el sistema inmunológico) para los niños de 9 años puede explicarse a través de algo más concreto (un soldado). Este es sólo uno ejemplo de los muchos que puede utilizar el profesor cuando trabaja con temas o textos expositivos y se busca que los estudiantes aprendan y comprendan el tema expuesto.

Una analogía puede entenderse como una comparación entre dos conceptos o temas; esta comparación se realiza buscando las semejanzas existentes entre ellos. De esta forma se pueden encontrar las similitudes y diferencias que permitan hacer la comparación. González (2004) en su libro Estrategias de comprensión lectora define las analogías como:

La transferencia de la estructura relacional de un campo temático conocido, denominado campo base o fuente, a otro en cierta medida similar; pero menos conocido, que se conoce como objetivo. Esta transferencia se consigue mediante un proceso de comparación y proyección (en el sentido cartográfico de reflejar en un mapa la realidad) por el que se detectan similitudes entre los dos sistemas: la estructura de una área de conocimiento familiar se proyecta dentro de otro campo de saber menos conocido, de esta forma se establecen correspondencias coherentes (uno a uno) entre dos ámbitos de conocimiento. (p. 86)

Por otro lado, las analogías se usan en la vida cotidiana, y en un lenguaje coloquial como proceso instruccional y, en este sentido, su finalidad es enseñar o explicar a alguien usando sencillas pero funcionales comparaciones. Es así como las analogías pueden desempeñar un papel muy importante en la comprensión de una situación o concepto.

Dentro del proceso de enseñanza-aprendizaje, el profesor utiliza este recurso para explicar contenidos científicos y tratar que de esta forma que sus alumnos aprehendan los conceptos e ideas que les enseña. 
Los niños y niñas de cuarto grado de educación primaria se encuentran entre la edad de 9 y 10 años, es decir, según el desarrollo cognitivo de Piaget, ellos se ubican en el estadio operacional concreto, factor indicador de que los alumnos aún requieren de situaciones concretas que los ayuden a asimilar el contenido o situación a comprender.

En la lectura, las analogías pueden tener la función de aproximar al estudiante de una manera más concreta al conocimiento; en este campo, las analogías se consideran como una estrategia pertinente que permite acercar al alumno a un conocimiento que para su desarrollo cognitivo resultaría por demás difícil. Así como lo manifiesta Ausubel cuando retoma a Inhelder y Piaget: “(...) los estudiantes que todavía no han sobrepasado la etapa concreta del desarrollo cognitivo son incapaces de incorporar de una manera significativa una relación entre dos o más abstracciones a sus estructuras cognitivas a menos que cuenten con la ventaja de una experiencia concreta y empírica actual o reciente" (Ausubel, 2002, p. 97).

Se trata de mostrar al alumno o alumna dos o más temas semejantes, donde, mediante las semejanzas entre ambos, se podrá realizar una comparación y proyección entre los dos campos de conocimiento, entendiendo que esta analogía o comparación de apoyo le permitirá encontrar ciertas semejanzas con las que podrá acercarse de manera más fácil al significado que debe adquirir, conceptos e ideas más concretos con los cuales podrá comprender otras proposiciones.

\section{Premisas básicas para la comprensión lectora: la información no visual y la estructura cognitiva}

Smith (1989) distingue dos tipos de información en la lectura, la información visual y la no visual; sostiene que:

(...) la información no visual se distingue fácilmente de la información visual: todo el tiempo la trae consigo el lector y no desaparece cuando las luces se apagan. Entre (sic) más información visual tenga un lector, menos información visual necesita. Mientras menos información no visual esté disponible detrás de los ojos, más información visual se requiere. (p. 17)

La información no visual es la que se encuentra detrás de los globos oculares, en la estructura cognitiva, llamada por otros conocimientos del mundo o conocimientos previos, (Sarmiento, 1995; Ausubel, 2002). Son aquellos conocimientos que se interrelacionan con un tema en particular y que se encuentran dentro de las estructuras cognitivas de cada persona o sujeto. Para Chadwick (2001) las estructuras cognitivas son:

(...) las representaciones organizadas de experiencias previas. Son relativamente permanentes y sirven como esquemas que funcionan activamente para filtrar, codificar, categorizar y evaluar la información que uno recibe en relación con alguna experiencia relevante. La idea principal aquí es que cuando captamos información, estamos constantemente organizándola en unidades con algún tipo de orden que llamamos "estructura". Generalmente la nueva información está asociada con información ya existente en estas estructuras, y a la vez esta información reorganiza o reestructura la información existente (p. 113).

La información que llega a través de los ojos y es llevada al cerebro tiene que encajar en una estructura cognitiva, la cual se encarga de codificar, relacionar e interpretar; cuando existen 
conocimientos almacenados en la memoria, entonces es más fácil la integración. Cuando se lee un texto, la estructura cognitiva se activa y comienza a buscar las nociones o ideas que se relacionan con lo que se está leyendo, de esta manera se genera una interacción entre el lector y el texto.

La diferencia que existe entre un lector que lee y comprende y uno que no lo hace es la estructura cognitiva. Una estructura cognitiva madura cuenta con información clara y disponible en relación con lo que se va a leer, de esta manera se puede llegar a comprender. La tesis principal de Smith (1989) es que cuando no se cuenta con la disposición y claridad de los conocimientos previos a la lectura, es decir, suficiente información no visual, la comprensión de esta se verá afectada drásticamente y producirá conflictos cognitivos.

Entre (sic) menos información no visual pueda emplear el lector, más difícil es la lectura. Ahora tenemos una razón para entender por qué la lectura puede ser tan difícil para los niños, independientemente de su habilidad real para leer. Ellos pueden tener poca información no visual relevante. (p. 18)

Para dicho autor, la comprensión lectora, o el proceso que implica este ejercicio, se lleva primero en un nivel perceptual con la información del texto; esa información explícita debe entrar en interacción con la que está detrás de los globos oculares; en esta interacción las ideas previas del lector servirán de anclaje a las ideas nuevas por aprender, por ello, es siempre importante que, antes de iniciar la lectura de un texto, se lleve a cabo esta parte de activación de los conocimientos previos, a través de preguntas, cuestionamientos, establecimiento de relaciones y ejercicios que activen las ideas de los niños.

Hasta aquí ha sido claro cuál es el problema al que se enfrentan los lectores principiantes o cualquier lector que no tiene información no visual. Es lógico que cuando no existe este tipo de conocimientos pre teóricos en el cerebro, lo que va a ocurrir es que se trate de memorizar el contenido del texto y retenerlo en la memoria, lo cual resulta prácticamente imposible, pues la demasiada información desconocida provocaría lo que Smith (1989) llama visión tubular.

La visión tubular es el resultado de tratar de procesar demasiada información visual. Todos los lectores pueden sentirse angustiados al experimentar una visión tubular cuando el material que están tratando de leer es desconocido, opaco o difícil. Los lectores principiantes son fuertes candidatos a tener visión tubular la mayor parte del tiempo, especialmente si los libros que supuestamente han de leer tienen poco sentido para ellos. La visión tubular, en otras palabras, es causada por una sobrecarga de información. (p. 42)

He ahí el fundamento y la importancia de los conocimientos previos que debe tener la persona cuando lee. Si no hay conocimientos previos sobre el tema, la lectura puede complicarse y será difícil que se establezca la conexión entre el lector y el texto, prácticamente estaría descontextualizado, y cuando eso sucede, como menciona Smith, es difícil integrar la información a la memoria a largo plazo, por lo que la memoria a corto plazo estará pronto sobrecargada con muchas palabras sin conexión y sin sentido para el lector.

Los conocimientos previos hoy se consideran como una de las premisas básicas para la comprensión. Los teóricos de la comprensión (Smith, 1989; Solé, 2001; Ausubel, 2002) sostienen que saber sobre lo que se va a leer es fundamental para el proceso de la comprensión y uno de las condicionantes más importantes que obstaculizan la asimilación y construcción de otros significados: sin conocimientos previos no se puede conferir significado y sentido a lo que se está leyendo. 


\section{La comprensión lectora desde el constructivismo}

Tres son los autores retomados en esta investigación, Piaget (1969), Vygotsky (1997) y Ausubel (2002). Las ideas que han aportado al proceso de aprendizaje permiten situarse en la postura de que el conocimiento es construido por el sujeto a través, definitivamente, de su desarrollo individual; pero también de su interacción con el medio ambiente, pues la maduración cognitiva se ve determinada por el contexto: es el medio sociocultural un factor determinante en la construcción del conocimiento.

\section{A) Teoría del desarrollo cognitivo de Piaget}

Para Piaget (1969), el sujeto comienza como un ser individual que progresivamente se convierte en social. El desarrollo está regido por la consolidación de estructuras mentales representativas del conocimiento, reguladas por los fundamentos biológicos del desarrollo, así como por el impacto de los factores de maduración.

Postuló que cada acto inteligente está caracterizado por el equilibrio de dos tendencias polares: la asimilación y la acomodación. Posteriormente, integra aspectos sociales que darían un giro radical a su teoría, y que la harían más funcional. Sostenía que los conflictos cognitivos eran la única fuente de desequilibrios y que esto, a su vez, propiciaría el aprendizaje y conocimiento. Es decir, el aprendizaje esta mediado por la búsqueda de equilibrio en una situación de conflicto.

Sostenía que el desarrollo dependía del factor genético o biológico y que la actividad de los niños era una construcción individual y aislada, que todo partía de un punto individual y privado, es decir, que el conocimiento sólo sería generado por el desarrollo genético y la maduración orgánica. Para esto propuso diferentes estadios de desarrollo: preoperacional, sensorio motriz, operaciones formales y operaciones concretas.

Desde esta perspectiva, es importante que en la enseñanza se tome en cuenta el desarrollo cognitivo del estudiante, pues con esto se asegura si es posible que el alumno lo pueda aprender o no. Piaget (1969) plantea que el ser humano pasa por diferentes estadios de desarrollo cognitivo con características particulares cada uno y en donde uno antecede al siguiente; pero es definitivamente la edad de la persona la que determinará la capacidad de entendimiento. En concreto, conforme se crece se va desarrollando una mejor manera de entender y aprender conceptos más abstractos y difíciles, así se llega a la etapa de las operaciones formales, donde es posible el desarrollo de la lógica y la abstracción.

En la lectura se debe considerar que tan "apto" está el niño para ese contenido o aprendizaje que se quiere transmitirle, pues es un hecho que si su desarrollo cognitivo no está acorde con lo que tiene que aprender, sería complicado y difícil que lo comprenda. De hecho, en la escuela el conocimiento y el aprendizaje tienen un orden sistemático y progresivo, que toma en cuenta los andamiajes propios del niño y de la niña.

Por lo tanto, los niños no pueden llegar a conocer sino aquellos objetos que son capaces de asimilar. Por ello, el concepto de asimilación usado por Piaget es fundamental para comprender el proceso de aprendizaje y el rol que juega la experiencia en las adquisiciones cognoscitivas del niño.

\section{B. El aprendizaje significativo de Ausubel}

Se ha definido y conceptualizado la comprensión lectora como un proceso constructivo, de interacción entre lector y texto, donde la mayoría de los autores que analizan el tema hacen referencia 
a la importancia de los conocimientos previos o de la información no visual. Así, se entiende por qué a los niños se les dificulta comprender textos con palabras y proposiciones desconocidas, cuando no cuentan con un referente empírico. Esto pasa hasta con los lectores "expertos" o "competentes", quienes, al no contar con un bagaje amplio, al leer tienen que hacerlo más de una vez, pues no hay información no visual que interactúe con el texto y toda la información visual que ve es nueva y su cerebro se debe esforzar más en decodificar y formar una estructura con significado que les ayude a comprender. En estos casos, solo podrían llegar a memorizar parte de la información leída, con la consecuencia de que el aprendizaje no resulta significativo y se olvida con facilidad.

Se ha versado, en párrafos anteriores, que cuando una persona lee, toma un papel activo y relaciona los conocimientos previos que posee con la información que le proporciona el texto. En esta interacción, la comprensión llega a darse con la construcción de significados nuevos y en este proceso es determinante la estructura cognitiva del sujeto para que haya aprendizaje significativo. A este respecto, Ausubel (2002) argumenta que:

(...) la esencia del proceso de aprendizaje significativo es que nuevas ideas expresadas de una manera simbólica (la tarea de aprendizaje) se relacionan de una manera no arbitraria y no literal con aquello que ya sabe el estudiante (su estructura cognitiva en relación con un campo particular) y que el producto de esta interacción activa e integradora es la aparición de un nuevo significado que refleja la naturaleza sustancial y denotativa de este producto interactivo. (p. 122)

Definitivamente, el papel activo del lector estará determinado por los conocimientos previos del mismo, por lo tanto, la comprensión lectora está sujeta a la estructura cognitiva, pues mientras más estructuras con significado posea el alumno, dispone de más recursos para interactuar con otras ideas mediante la lectura, mayor posibilidad tendrá de construir otras más complejas, facilitándose, de esta manera, la comprensión lectora y el aprendizaje significativo.

Para el autor, comprender una frase implica: primero, percibir el significado proposicional potencial que comunica (comprender los significados denotativos y las funciones sintácticas de las palabras componentes); en segundo lugar, incorporar este significado potencial percibido a la estructura cognitiva ya existente.

Agrega que cuando los niños dominan el código sintáctico y un vocabulario básico, los únicos aspectos cognitivos para comprender una frase están asociados con relacionar las ideas que contiene con una proposición (idea) oportuna ya existente en la estructura cognitiva. Los significados denotativos y las funciones sintácticas de las palabras componentes ya son significativos y, en consecuencia, se pueden captar mediante la percepción. Incluso la comprensión del propio significado proposicional se convierte en un proceso puramente perceptivo cuando el mensaje se repite una o más veces.

Hasta aquí se ha visto que existe una relación muy estrecha entre la comprensión de textos y la estructura cognitiva o los conocimientos previos; pero, ¿cuál es el papel del uso de analogías en la comprensión lectora y cuál es la relación que guarda con los conocimientos previos o la estructura cognitiva? En situaciones reales ocurre que muchas veces las personas no tienen conocimientos previos pertinentes sobre el tema, o su estructura cognitiva no es la más "madura" para poder comprender la información visual-impresa o auditiva. Entonces, cuando esa persona se convierte en pasiva, es decir, que lo que leyó o escuchó no fue lo suficientemente significativo para poder comprenderlo, el uso de analogías resulta pertinente para acercar, de manera más concreta, al lector mediante situaciones que lo ayuden a relacionar la información nueva con otra que ya posee, la cual le va a servir de base para ir construyendo esa nueva idea con significado que aún no concretaba. 


\section{C) La perspectiva sociocultural de Vigotsky}

La línea cultural establecida por este autor fundamenta el medio social y cultural es uno de los factores más determinantes en el conocimiento o cognición de una persona. Plantea que es la sociedad y la interacción con los otros lo que permitirá el desarrollo cognitivo, por lo que, sostiene, no es sólo el desarrollo biológico o natural el que permite aprender, conocer o comprender. Establece que dentro del proceso de aprendizaje es importante la ayuda que se recibe de los demás: los niños y las niñas aprenden con la ayuda de sus iguales o con la ayuda de sus padres y docentes. Vigotsky, en sus estudios teóricos sobre el aprendizaje, analiza la Zona de Desarrollo Próximo (ZDP). La ZDP es el área que existe entre la ejecución espontánea que realiza el niño o el adolescente utilizando sus propios recursos y el nivel que puede alcanzar cuando recibe apoyo externo.

De esta manera, el apoyo que el alumno/a debe recibir del profesor, corresponde a una ayuda en su aprendizaje, en situaciones que por sí solo no podría resolver. El objetivo consiste en propiciar el pensamiento y razonamiento propio, a través de situaciones pertinentes que le permitan dar un paso entre lo que sabe y lo que está por aprender.

En la teoría sociocultural, los instrumentos culturales en los que está inmerso el alumno/a juegan un papel en el desarrollo cognitivo: los maestros, la familia y la comunidad deben establecer una relación mutua en la formación del sujeto.

Para decirlo con Vigotsky (1997), la zona de desarrollo proximal es la distancia entre el nivel de desarrollo real del niño, tal y como puede ser determinada a partir de la resolución independiente de problemas, y el nivel más elevado de desarrollo potencial, tal y como es determinado bajo la guía del adulto o tutor educativo. En este sentido, el papel explícito del profesor y profesora es el de provocar, en el alumno/a, avances que no sucederían de manera espontánea, que no podrían alcanzar por sí solos.

En este sentido y concretando el uso de la ZDP en la utilización de analogías en la comprensión lectora, es precisamente cuando se trata de ayudar al alumno/a mediante la relación de dos contenidos: uno es lo que él o ella sabe o conoce (lo que ellos pueden hacer por sí mismos) y otro, cuando necesita la ayuda o apoyo del profesor y este se vale del recurso analógico como herramienta de instrucción o explicación para que pueda entender otros conceptos que por sí sólo, los que, sin uso de otros recursos, no lograría comprender.

Vigotsky (1997) argumentaba que los procesos psicológicos deben estudiarse en su desarrollo. Definía el desarrollo en términos de saltos revolucionarios fundamentales más que como incrementos cuantitativos constantes. A partir de cierto momento del desarrollo, las fuerzas biológicas no pueden ser consideradas como la única ni incluso la principal fuerza del cambio. En ese momento, hay una reorganización fundamental de las fuerzas del desarrollo, así como una necesidad de su correspondiente reorganización de los principios explicativos del sistema.

El desarrollo natural produce funciones con formas primarias, mientras que el desarrollo cultural transforma los procesos elementales en procesos superiores.

Vigotsky concibe la dinámica del dominio ontogénico como el desarrollo definido en términos de la relación entre las fuerzas naturales y culturales. Aunque las premisas teóricas del autor sugieren que las dos líneas del desarrollo convergen en una relación de transformación mutua, sus propuestas concretas y su invaluación empírica revelan, persistentemente, otra interpretación que supone una influencia unidireccional, en concreto: la inteligencia práctica de la línea natural de desarrollo se transforma como resultado de entrar en contacto con aspectos de la línea social. 


\section{Metodología}

La investigación se enmarcó en un diseño descriptivo-cualitativo. El objetivo general fue describir de qué manera el uso de analogías como estrategia favorece la comprensión lectora en alumnos de cuarto grado de primaria, a través de una encuesta etnográfica, un cuestionario y un modelo pedagógico para el uso de analogías en la lectura de textos expositivos.

El diseño metodológico propuesto para esta investigación se desarrolló en tres fases.

Primeramente se hizo un estudio etnográfico de la comunidad para recoger datos generales del contexto, que sirvieron como marco de referencia.

La encuesta se realizó para conocer el marco general del contexto donde vivían los estudiantes con los cuales se realizó esta investigación. En esta fase se conocieron aspectos sociales, económicos y culturales. Se formularon 16 preguntas sobre el nivel de escolaridad, ingreso familiar, ocupación, recreación, características generales del medio social, cultural y ambiental que mostraran un panorama del contexto donde el alumno/a se desenvuelve.

Posteriormente se hizo un estudio diagnóstico a través de un cuestionario propiamente educativo con preguntas cerradas, para tener referentes empíricos sobre la comprensión y el uso de analogías en el grupo de trabajo. El cuestionario se construyó con base en las categorías de análisis: comprensión lectora y analogías. Se formuló con 21 preguntas sobre el proceso que siguen en la lectura y sobre el uso de analogías.

Finalmente, se aplicó un instrumento pedagógico-cualitativo, en el cual se diseña un Modelo Pedagógico para uso de Analogías en la Lectura, cuyo objetivo fue identificar de qué manera el uso de analogías puede favorecer la comprensión lectora en alumnos de cuarto grado de educación básica primaria. Este instrumento se estructuró y se aplicó en cuatro fases: Primero se presentó al alumno el proceso metamórfico de la mariposa, de hecho conocido por ellos, pues es algo observable en el medio ambiente. Posteriormente se introduce al tema de la evolución humana, preguntando lo que ellos conocen al respecto. Se hizo la lectura de la evolución del ser humano, contenido que se esperaba pudieran comprender. Después se establecieron las semejanzas entre el proceso metamórfico de la mariposa y la evolución del ser humano (esto se realizó con ayuda del instrumento, donde, en dos columnas se organizó la analogía, del lado izquierdo se estableció, con texto e imágenes, la metamorfosis de una mariposa y del lado derecho de la página, el proceso de evolución del ser humano). Finalmente se realizó la comparación, donde se establecieron las semejanzas y las diferencias entre los dos campos de conocimientos. Para concluir se dio a los estudiantes una retroalimentación del tema por parte del docente.

Para evaluar el impacto de las analogías como estrategia en la promoción de la comprensión lectora, se construyó un cuestionario con 15 preguntas sobre el tema de la evolución del ser humano, cuyo objetivo fue medir su grado de comprensión. Una vez llevado a cabo el proceso de comparación (analogía), el cuestionario se construyó con las categorías de análisis propuestas en la investigación: comprensión lectora y analogías.

Los resultados de los instrumentos se vaciaron en Excel, se obtuvieron frecuencias de la encuesta y del cuestionario diagnóstico. Posteriormente, el Modelo Pedagógico para el Uso de Analogías fue evaluado con un análisis de contenido, según (Krippendorff, 1990). 


\section{Resultados}

\section{A) Marco de referencia: la comunidad}

Conocer las características del medio social donde viven los participantes fue la primera fase de la investigación, esta se realizó mediante una encuesta a los padres de familia que arrojó datos importantes como el nivel de estudio, ingreso familiar, ocupación, actividades económicas, actividades recreativas y culturales, así como los principales servicios públicos que existen en la comunidad.

La comunidad donde se realizó el estudio es rural con 1400 habitantes, sólo cuenta con un preescolar y una primaria, esta última atiende a 210 estudiantes. Su flora y fauna aún es diversa, se pueden encontrar animales como caballos, borregos, chivos, patos, gallinas, chapulines, vacas, burros, cerdos, gatos y perros. Dentro de los árboles hay pirules, eucaliptos, alcanfor, duraznos, chayotes, olivos, capulín, tejocotes, girasol, maguey, rosales, lirios, alcanfores, nopales, zarzamoras y biznagas.

No cuenta con secundaria ni preparatoria, por lo cual, cuando los niños y las niñas de la comunidad terminan la primaria tienen que acudir al municipio donde encuentran la oportunidad de continuar estudiando. El ingreso mensual aproximado de las familias es de 1500 pesos y suelen ocuparlo en la comida y en la educación. Los integrantes de la familia van de 4 hasta 7 y como máximo 8 personas.

De los 25 padres de familia, 11 cuentan con primaria, 10 con secundaria y sólo 4 concluyeron o tienen estudios técnicos o de preparatoria. De lo que se desprende que el núcleo familiar de los participantes y las participantes no tiene un estatus educativo alto, con consecuencias que se traducen en no saber cómo apoyar a sus hijos e hijas en las actividades extraescolares o, simplemente, carecen de un medio alfabetizador que favorezca el desarrollo de actitudes y aptitudes para la lectura y otras actividades educativas.

\section{B) Diagnóstico de los participantes y las participantes}

A continuación se detallan los resultados encontrados en una primera fase de la investigación, resultados que nos acercaron de manera concreta a la situación en que se torna la lectura, se describen por dimensiones o categorías de análisis:

Cuadro 1

Parámetros establecidos para la interpretación de resultados del diagnóstico y del Modelo Pedagógico para el uso de analogías en la lectura, en términos de porcentaje

\begin{tabular}{|c|c|c|c|c|c|}
\hline Muy bajo & Bajo & Medio & Alto & Muy alto & Categorías de análisis \\
\hline $1-20 \%$ & $21-40 \%$ & $41-60 \%$ & $61-80 \%$ & $81-100 \%$ & Comprensión lectora y uso de analogías \\
\hline
\end{tabular}

Estos parámetros miden las aptitudes del grupo hacia la lectura y el uso de analogías. La muestra total la constituyen 27 alumnos y alumnas de $4^{\circ}$ grado de educación primaria. 
De un total de 27 alumnos (14 mujeres y 13 hombres) a los cuales se les aplicó el cuestionario de diagnóstico, el 25\% cuando lee lo hace para conocer más; el 22\% del estudiantado, para informarse; el 37\%, para contestar preguntas; el 14\%, para practicar la lectura en voz alta. No hubo alumnos que no se plantearan objetivos de lectura. La lectura en voz alta es un ejercicio poco practicado, considerado como aspecto formal de la misma, pues generalmente dentro este ejercicio se media la rapidez, la dicción y la fluidez. Lo ideal sería que todos los niños y las niñas leyeran para conocer más o para informarse, y sólo ocurre con el $47 \%$ del total. La lectura, entonces, se vuelve una actividad mecanizada, donde los cuestionarios aún siguen siendo usados como estrategia para el aprendizaje.

\section{b) Predicción y anticipación:}

El $62 \%$ de la población de estudiantes realiza predicciones y anticipa el contenido del texto, pues revisa las imágenes, el titulo y los subtítulos para saber de qué va a tratar la lectura. Esto significa que la mayoría de los estudiantes se ubica en un nivel bueno en aptitudes hacia la lectura, pues llevan a cabo la estrategia de predicción y anticipación. El 38\% restante no lleva a cabo esta estrategia como parte del proceso y suele irse directo a la información, con lo que se ubica en un nivel bajo.

Los resultados sobre el uso de la anticipación como estrategia para la comprensión lectora fueron que: el $59 \%$ al revisar las imágenes, el título y los subtítulos del texto pueden anticipar el contenido, el $37 \%$ solo a veces y el $4 \%$ no puede.

El 85\% de la población del estudio cuando no comprende la lectura se detiene y vuelve a leer el párrafo, el 11\% investiga más en otro libro y el $4 \%$ deja de leer. Estos datos indican que la mayoría de los estudiantes busca comprender la lectura aún cuando tenga que regresar a ella. La metacognición supone que los alumnos deben conocer su propio proceso lector, es decir, darse cuenta de sus fallos y aciertos en la lectura. El detenerse y volver a leer el párrafo implica un análisis metacognitivo, pues se tiene conciencia de cuándo se ha comprendido y cuándo no. El grupo puede ubicarse en un nivel muy alto respecto a esta estrategia.

\section{c) Conocimientos previos:}

Respecto a este indicador, las preguntas se limitaron a conocer si el alumno y alumna utiliza sus conocimientos previos (en adelante denominado c. p.) en la lectura y también saber qué es lo que sucede desde su punto de vista cuando no saben sobre lo que van a leer. Los resultados en torno a esto, muestran que la mitad de la población piensa que si tiene conocimientos previos, es decir, estos alumnos y alumnas saben de esta estrategia y la aplican cada vez que tienen que leer un texto. El 33\% de los estudiantes, dice que cuando no tiene c.p. no entiende lo que dice el texto o no puede saber de lo que tratará la lectura, y el 37\% se preguntan sobre lo que va a leer. Con esta información obtenida se deduce que se muestran como lectores activos. Los resultados sólo confirman, desde la perspectiva de los participantes, la relevancia que juegan los c.p. en la lectura para poder llegar a comprender un texto.

El $48 \%$ suele tener conocimientos previos del tema cuando lee algún texto. El $7 \%$ no tiene y el $45 \%$ solo a veces. El $22 \%$ cuando lee aporta los c. p. El 22\% la mayoría de las veces, el $51 \%$ 
muy pocas veces y el $4 \%$ no lo hace. Estos resultados permiten concluir que los niños, en pocas situaciones o eventos de lectura, tienen c.p. sobre el tema; en algún momento, parece que sólo la mitad $(50 \%)$ cuenta con referentes antes de la lectura. Sin embargo, estos resultados pueden resultar positivos para la propuesta de investigación, ya que el uso de analogías constituye un referente práctico o concreto que puede coadyuvar a que los alumnos, aún cuando no tengan c. p., puedan tener un aprendizaje significativo.

\section{d) Inferencias:}

De acuerdo con la pregunta planteada, el $84 \%$ de los sujetos deduce nueva información y obtiene conclusiones correctas sobre un tema. El 16\% no lo hace. A partir de párrafos los sujetos obtienen la idea central o más importante del mismo, de esta forma se determinó quiénes podían obtener la conclusión general o la idea central de un texto. Por ejemplo: Las abejas recolectan la resina que está en la corteza de algunos árboles, conforme la chupan con la lengua y la trompa, la guardan en ocho bolsitas que llevan en el abdomen. Cuando las bolsitas están llenas, llevan la resina a su boca y la mastican hasta que cuaja y se forma una cera. Con esta van levantando pequeñas celdas hexagonales una tras otra, así poco a poco construyen el panal. ¿La idea central del párrafo anterior es? ${ }^{2}$

El 59\% comprende la idea o el contenido central de un texto, el $41 \%$ de la muestra no lo hace. El 74\% elabora conclusiones generales o globales a partir de un texto, el $26 \%$ no lo hace.

Con respecto a que si el alumno puede relacionar, comparar, proyectar y obtener inferencias correctas a partir de textos analógicos, los resultados indican que existe un pensamiento analógico en los niños de 9 y 10 años de edad. Este indicador se pudo observar mediante analogías, deducciones e inferencias de acuerdo con el nivel y desarrollo de los participantes, por ejemplo: si leíste un tema sobre los anfibios y dices que los sapos son anfibios porque pueden vivir dentro y fuera del agua, ¿entonces las ranas?

El 70\% puede transferir información de un campo conocido a uno menos conocido. El 30\% no lo hace, con esto se determina que el grupo se ubica en un nivel bueno y tiene un pensamiento inferencial adecuado, además de que un porcentaje alto logra hacer deducciones correctas.

El 30\% distingue la similitud entre dos situaciones analógicas, el $30 \%$ no puede y el $40 \%$ restante solo a veces lo hace.

El $74 \%$ proyecta la información del campo conocido al campo de conocimiento menos conocido, el $26 \%$ no lo hace. Fueron 2 preguntas con este indicador y los resultados son parecidos, $85 \%$ sí y el $15 \%$ no.

\section{e) Relación y comparación:}

Para llegar a estos resultados se plantearon preguntas como: se dice que un pato es un animal ovíparo, pues nace del huevo, ¿entonces una tortuga?; los idiomas o lenguas fueron inventados por el hombre a través de la historia y por la necesidad de comunicarse. El náhuatl es una len-

\footnotetext{
La respuesta a esta pregunta es cómo las abejas construyen el panal. Por los resultados se deduce que los participantes pueden obtener conclusiones generales o globales. En el cuestionario corresponde a la pregunta 9, categoría de análisis comprensión lectora, indicador inferencias.

3 La inferencia a esta pregunta es que por lo tanto las ranas son anfibios, ya que también pueden vivir dentro y fuera del agua. Este es un ejemplo de preguntas que permiten obtener inferencias a partir de la comparación y deducción. Esta pregunta corresponde al número 11, categoría de análisis comprensión lectora, indicador inferencias.
} 
gua, ¿entonces? $?^{4}$ Otro caso es la pregunta 14 del cuestionario: Todas las zonas arqueológicas de México fueron hechas por el ser humano, el Tajín es una zona arqueológica, ¿entonces?

El 26\% sí puede relacionar la información que le proporciona un texto con la que conocen. El $15 \%$ no puede y el $59 \%$ sólo a veces.

El 44\% relaciona y compara la información de un tema conocido con otro menos conocido y distingue las semejanzas analógicas entre dos campos de conocimiento, el 56\% de los sujetos no lo hace.

f) Tipos de texto:

Al $67 \%$ de los sujetos prefieren leer cuentos y leyendas, es decir, textos narrativos; al 15\% le gusta leer poesía, al $7 \%$ noticias y al $11 \%$ le gusta leer textos científicos.

De esto se puede concluir que a los niños y las niñas no les gusta leer textos informativos o expositivos, con demasiada información que implique comprensión, análisis, aprendizaje de hechos y sucesos sobre un tema, los cuales, a diferencia de los narrativos como los cuentos y las leyendas que se leen por diversión y que además te entretienen y divierten. Los textos expositivos requieren no sólo su capacidad para descifrar, sino de poner en práctica estrategias que los ayuden a comprender, las que, para muchos niños, constituyen un proceso complejo y difícil de llevar a cabo.

\section{C) Interpretación de los resultados del diagnóstico}

Los resultados que arrojó el cuestionario diagnóstico nos permitió conocer diferentes aspectos del objeto de estudio, la lectura, y de las analogías como estrategia, de esta manera que se llegaron a inferir ciertas conclusiones derivadas de un análisis de cada uno de los indicadores y que se concretan en lo siguiente:

a) La mayoría de los alumnos siguen estrategias cognitivas en la lectura, las que los ayudan a comprender un texto y muestran características de los lectores activos como:

Se plantean objetivos de lectura. Hacen predicciones y anticipan el contenido del texto. La mayoría de los sujetos puede predecir y anticipar la información a partir de las imágenes, los títulos y los subtítulos. Los sujetos dicen ser conscientes que cuando no comprenden, se detienen y vuelven a leer el párrafo.

La mitad de la población toma en cuenta sus conocimientos previos. Por otro lado dicen que cuando no tienen conocimientos previos no comprenden la lectura o no saben de qué va a tratar.

b) La mayoría tiene un pensamiento analógico, pues logran comparar información de campos de conocimientos similares, deducen nueva información y logran obtener inferencias correctas.

c) Desde esta premisa se fundamenta la aplicación de la segunda fase de la investigación, puesto que los sujetos han demostrado en la medición de la variable analogías que sí

Estas preguntas corresponden a la categoría de análisis analogías y a la dimensión similaridad y proyección. Mediante esto se observó quienes podían obtener una inferencia correcta a partir de las similitudes y de la proyección que existe en las analogías presentadas. Estas preguntas corresponden a la 19 y 20 del cuestionario. Las inferencias correctas fueron: es ovípara en el caso del pato y la inventó el ser humano en el caso de los idiomas o lenguas. 
tienen los elementos para poder relacionar, comparar, proyectar, trasferir y generar inferencias correctas a partir de dos situaciones de conocimientos analógicas.

\section{D) Resultados de la aplicación del Modelo pedagógico para el uso de analogías \\ a) Comprensión lectora}

Objetivos de lectura: De los 27 participantes con los cuales se trabajó el Modelo pedagógico para el uso de analogías en la lectura, el 73\% del grupo indica que su objetivo de lectura es para saber sobre la evolución del ser humano. El 11,5\% para conocer más sobre el tema y 15,3\% indica que leerán para aprender.

Predicción y anticipación: El 84,6\% de los sujetos pueden anticipar o predecir el contenido de la lectura al señalar como temática las diferentes etapas evolutivas del ser humano a través de millones de años. El 15,3\% no aplica esta estrategia al elegir otras opciones.

Conocimientos previos: El 69,2\% del grupo señala tener conocimientos previos y la mayoría hace referencia a que el ser humano viene del mono, de lo que se desprende que sí han escuchado sobre este tema. El 30.7\% no contestó a la pregunta, por lo tanto, se considera que no tienen conocimientos previos. Por lo anterior, concluimos que dentro de la aplicación del Modelo pedagógico para el uso de analogías, los estudiantes pudieron interactuar con el texto, profundizar en lo que ellos ya conocían y, por consiguiente, modificar sus esquemas mentales respecto de la evolución del ser humano.

Inferencias: El 73\% del grupo en el indicador de inferencias logró obtener una conclusión correcta a partir de la lectura, la cual fue: los cambios en el ser humano fueron físicos y mentales.

El 50\% del grupo entendió la idea central del texto: el ser humano evolucionó a través del tiempo pasando por diferentes etapas evolutivas que lo iban distinguiendo y haciendo diferente a los anteriores.

El 69\% de los sujetos planteó la hipótesis correcta, pues menciona que el estudio de los restos homínidos encontrados sirve para explicar cómo el ser humano fue evolucionando a través del tiempo.

El 61,5\% de la muestra dio una respuesta acertada a esta pregunta, que resolvía si los sujetos podían obtener una inferencia correcta después de haber hecho la lectura. En opinión de 16 sujetos, el último cambio en la evolución del ser humano fue su forma de pensar; 6 dicen que fue su habilidad y 4 que su físico.

El 65,3\% de la muestra, a partir de la lectura, plantea que la evolución del ser humano se dio a través de millones de años. El 34,6\% del estudiantado plantea que por etapas. Ambas respuestas son correctas, puesto que giran en torno al tiempo evolutivo. Sólo 2 sujetos dijeron que en un solo cambio.

La finalidad u objetivo es que los sujetos comprendieran el término evolución. Más de la mitad $(53,8 \%)$ de la muestra dijo que evolución significa cambio; el 38,4 \% de la misma menciona que implica un proceso y sólo el 7,6\% del grupo contesta que evolución es permanencia. Estos datos permiten concluir que el concepto evolución fue comprendido por la mayoría de los estudiantes.

\section{b) Analogías}

Transferencia: El 84\% puede observar que en cada ciclo de la mariposa se observa una relación, en cada una se da el cambio como denominador común y esto, a su vez, es llevado o 
traspolado a las etapas de evolución del ser humano, mencionando que en cada una hay diferencias muy notorias.

Relación y comparación: El 60\% de los sujetos sabe cuál es la relación que existe entre la metamorfosis de una mariposa y la evolución del ser humano, pues logran comparar y relacionar las etapas de cada ser vivo. El $27 \%$ toma en cuenta las etapas evolutivas del ser humano y las compara con las etapas de cambio de la mariposa, logrando ver que en ambos procesos hay cambios.

Similitud: El 84,6 \% de la muestra logra distinguir la similitud y, por tanto, compara ambos procesos en las semejanzas que tienen e indican que el proceso evolutivo del ser humano y el proceso metamórfico de la mariposa son, ambos, procesos de cambio; el 11,6\% menciona que ambos evolucionan, pero no cambian, y el 3,8\% menciona que no hay cambio ni evolución.

Proyección: Nuevamente, en este indicador, 18 sujetos (70\% del grupo) del total afirman que en la evolución del ser humano cada etapa es diferente a la anterior, es decir, que hay un denominador común, una relación y este nuevamente es el cambio.

\section{Discusión teórica}

\section{A) Comprensión lectora}

Objetivos de lectura: El aprendizaje significativo puede darse en cuanto las personas tomen una actitud positiva hacia lo que van a leer, es decir, se trata de encontrar un sentido o finalidad a la lectura. Esta actitud está mediada por lo que quiere lograr el lector, por el objetivo. Los resultados en relación con los objetivos se focalizan en torno a: saber sobre la evolución del ser humano, conocer más sobre el tema y aprender. En este tenor, Solé (2001) indica que los lectores pueden establecer diferentes clases de objetivos dependiendo de lo que quieren lograr en la lectura, de tal manera que la lectura puede definirse en términos de lo que quiere el lector. Así se puede decir que se lee para contestar un cuestionario, por diversión, para encontrar una información específica, etc.

Todos los sujetos se plantearon diferentes objetivos de lectura, es decir, tenían un por qué o para qué del leer. Ahí se encuentra parte del interés por leer, conocer el "para qué" es fundamental, pues es la guía que dirige el proceso. Uno de los objetivos que se plantearon los y las participantes ha sido el de contestar preguntas, es decir, una actividad que permea la lectura en los salones de clase es la de contestar preguntas, por lo que la lectura se convierte en una actividad consistente en la búsqueda de respuestas.

Predicción y anticipación: Otros elementos que están presentes en el proceso de la lectura son la predicción y la anticipación. Al anticipar el contenido mediante la observación de títulos y subtítulos o de las imágenes, se genera una activación de la estructura cognitiva que indicará si se cuenta con los conocimientos previos o no, esto es, anticiparse es preparase para lo que puede venir y determinar si se cuenta con conocimientos pre teóricos pertinentes del tema o lo que Ausubel (2002) llamaría conocimientos pertinentes.

Los sujetos manejan la predicción como estrategia previa para la comprensión de la lectura, esto es, pueden dilucidar el contenido de un tema. En este sentido, Solé argumenta que la predicción es fundamental para la comprensión. Tal como Smith (1989), concibe la predicción como la eliminación de posibles alternativas, discriminar de entre las no viables aquellas que resultan coherentes con el tema.

Conocimientos previos: Según Ausubel (2002), en la comprensión del material visualimpreso o auditivo, respecto de los conocimiento previos y de la estructura cognitiva, cuantas 
más ideas se tengan sobre el tema estas podrán interactuar con la nueva información que se va a aprender. Como el mismo lo menciona, estas servirán de anclaje en la restructuración de los esquemas mentales del sujeto.

Los conocimientos previos convergen en lo que Vigotsky (1997) define como Zona de desarrollo próximo (Z.D.P.), al considerar que estos serán la base para la adquisición de nuevos significados cuando se asocian al marco conceptual preexistente del sujeto. Cuando el alumno cuenta con experiencias concretas previas, resulta para él más sencillo actuar por sí mismo, sea en la lectura o en la solución de problemas.

Por lo anterior, se puede inferir que los conocimientos previos estuvieron presentes en la mayoría de los sujetos, logrando así una interacción y aportación al tema sobre el cual leyeron.

Inferencias: Para medir esta dimensión (inferencias) se toman en cuenta indicadores como: planteamiento de hipótesis apoyadas en la información del texto, comprensión de la idea o el contenido central de un texto, deducción de nueva información y elaboración conclusiones generales o globales; todo por parte del alumnado. Solé (2001) señala que las estrategias para la lectura de comprensión que se trabajen con los sujetos no deben soslayar esta característica, ya que mediante las inferencias se pueden llegar a obtener conclusiones generales o globales de la lectura, además de que es un recurso para la generación de ideas nuevas.

\section{B) Analogías}

Transferencia: Desde la definición que se ha dado de analogías, la transferencia se observa mediante un proceso de comparación y proyección. Los teóricos de la comprensión sostienen que, no siempre que el sujeto cuente con el conocimiento, esto va a indicar que hay comprensión. Argumentan que esta se va a dar en cuanto tengan la capacidad de transferir esa información a otros contextos o situaciones. Entonces, la transferencia juega un papel relevante en la comprensión. En las analogías se trata de transferir la información del campo fuente, al campo objetivo y, mediante esto, se puedan trasladar las estructuras semánticas entre los dos campos de conocimiento. Como ha sucedido con los sujetos del grupo de trabajo para esta investigación, cuando se les pregunta cuál es la relación que existe entre las etapas del proceso metamórfico de una mariposa y la evolución del hombre.

Los resultados al respecto indican que la transferencia entre dos campos de conocimientos distintos es posible en cuanto se conozcan claramente las semejanzas y las diferencias que existen entre los mismos. Transferir, en este sentido, es llevar el significado mismo de un tema y hacerlo encajar en otro en cierta medida similar.

Relación y comparación: Mediante las semejanzas que existan se van a generar razonamientos y conductas que se fundamentarán en las características que existan entre los dos campos de conocimiento similares. Estas van a llevar a la modificación de los esquemas mentales de la persona que realice la comparación. Cuando se habla de esta modificación de esquemas mentales, se refiere al aprendizaje significativo de Ausubel (2002), quien dice que partimos de lo que el alumno o el sujeto ya sabe o conoce, es decir, de los conocimientos previos. Posterior a esto, viene la relación de esos conocimientos con los que le puede aportar la lectura y, mediante una transacción flexible o una interacción propiamente dicha, se van a generar nuevas ideas con significado, que nuevamente podrían ser modificadas o reestructuradas, originando nuevos conocimientos o a la ampliación de los viejos.

En este orden se puede plantear que la analogía permite este tipo de comparación, con la cual se pudieron establecer las semejanzas y las diferencias entre los dos campos de conocimiento: por un lado el proceso metamórfico de la mariposa considerado como el campo base o fuente, y, por otro, la evolución del ser humano considerado como el campo objetivo. Así, las etapas de cambio de la 
mariposa, huevo, oruga, capullo y mariposa, se comparan con las etapas evolutivas del ser humano, australopitecos, homo hábilis, homo herectus y homo sapiens. De esta manera, se pueden establecer las semejanzas entre ambos campos de conocimientos, tal como ha sucedido en las etapas de cambio de la mariposa, el ser humano, también evolucionó, pasando por diferentes periodos evolutivos que lo iban haciendo diferente no solo en el aspecto físico, sino también, en su pensamiento.

Comparar estos campos de conocimiento permitió a los alumnos y alumnas profundizar en el tema de la evolución del ser humano, conocerlo y comprender cómo se dio la evolución humana.

Similitud y proyección: Esta característica de las analogías como estrategia es el fundamento de las conductas y razonamientos generados por el proceso de comparación. Una vez que los sujetos pueden distinguir las similitudes o semejanzas existentes entre los dos campos de conocimiento, están listos para proyectar la información e integrarla a su estructura cognitiva, mediante una comparación de la estructura semántica. En este caso, los sujetos lograron encontrar las similitudes en los dos campos de conocimiento presentados: la metamorfosis de una mariposa y la evolución del hombre.

Esquemas interpretativos del uso de analogías en la comprensión lectora

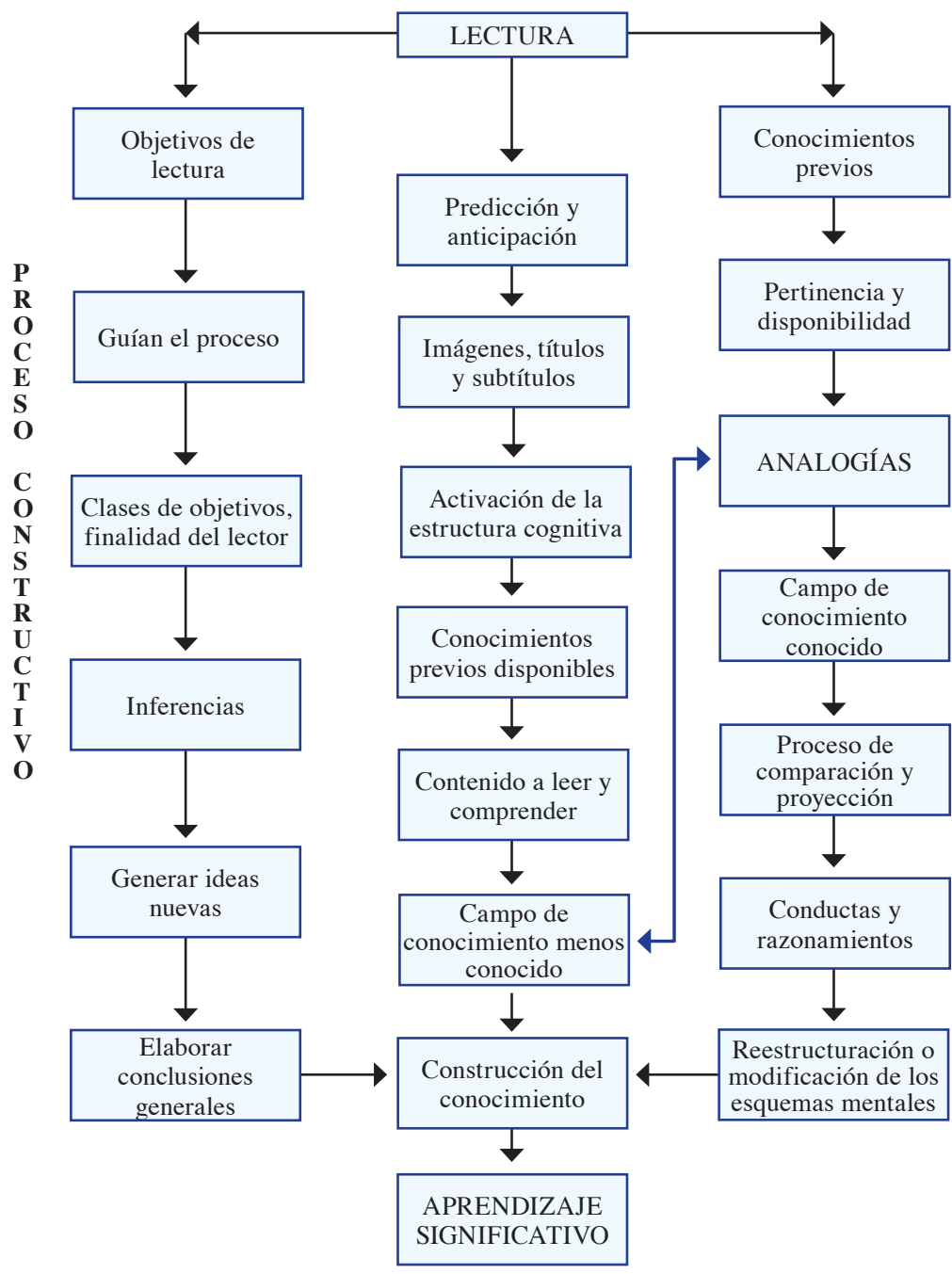

El proceso de la lectura requiere de la integración de varios aspectos fundamentales para que exista la comprensión. En este proceso convergen de manera integral los objetivos de lectura, la predicción, la anticipación, los conocimientos previos y las inferencias. Se puede destacar el uso de analogías como estrategia paralela al proceso, al relacionar o comparar lo leído con información similar de otro campo de conocimiento.

Figura 2

Proceso global de la lectura y su relación con las analogías. (Elaboración propia). 


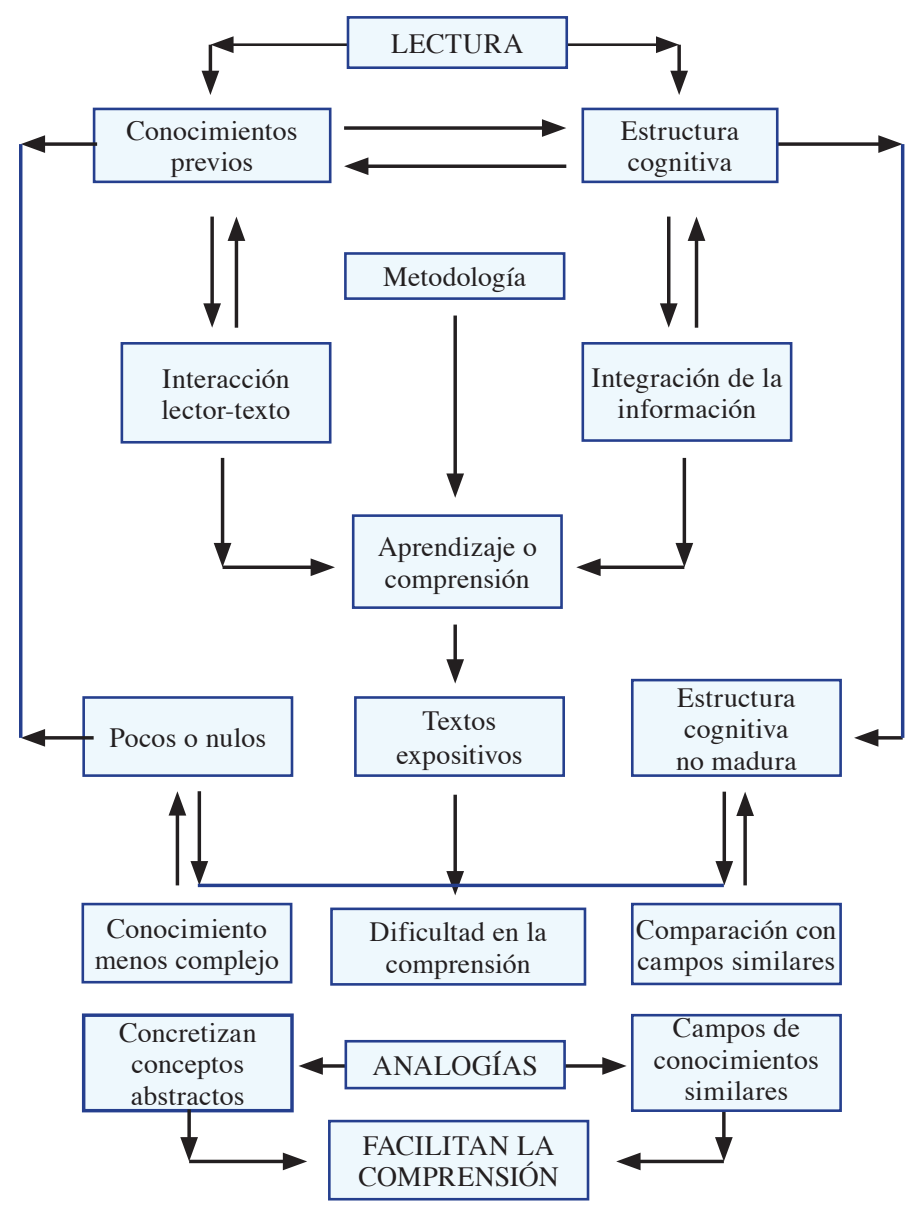

La lectura es un proceso que parte de los conocimientos previos y la estructura cognitiva, requiere de la interacción entre el lector y el texto y de una integración de la información, estos aspectos mantienen una relación mutua y confluyen en la comprensión. Se manifiesta que cuando uno de estos elementos falla la comprensión se dificulta y es ahí donde las analogías cumplen su cometido y facilitan la comprensión.

Figura 3

Factores que intervienen en la lectura y su relación con las analogías. (Elaboración propia).

\section{Conclusiones}

La comprensión lectora es un proceso que se presenta en el nivel de la estructura cognitiva de los sujetos, es un proceso de construcción de significados en el cual intervienen factores como: los conocimientos previos, los objetivos de la lectura, la predicción, la anticipación, la motivación, el contexto, las inferencias, la deducción de nueva información y la elaboración de conclusiones generales a partir de la información.

De los aspectos anteriores, es importante precisar que una de las condicionantes que más pueden influir en la comprensión, de acuerdo con Ausubel (2002) y Smith (1989) son los conocimientos previos o la estructura cognitiva. Estos autores sostienen que la comprensión se puede dificultar al no tener una estructura cognitiva "madura". La inmadurez de esta estructura puede traer consigo una mala comprensión o un aprendizaje totalmente memorístico.

El uso de analogías favorece la comprensión lectora, al acercar, de manera concreta, conceptos difíciles para los lectores. Hacen clara la información, mejorando la codificación y organización del conocimiento. Con las analogías se relaciona y compara información de un tema conocido para los lectores con uno menos conocido. Esta comparación requiere de procesos cognitivos para encontrar 
semejanzas o similitudes entre ambos campos de conocimiento que generen la modificación de conductas y razonamientos, llevando a la reestructuración del marco conceptual y de la estructura cognitiva. De esta manera, si el lector no cuenta con conocimientos previos suficientes antes de la lectura, la analogía le permitirá activarlos y concretar ideas que para él son difíciles de comprender.

El uso de analogías se establece dentro del proceso metodológico de la lectura como una estrategia cognitiva paralela a todo el proceso. Es decir, puede ser aplicada como una estrategia junto con el proceso: antes, durante y después de la lectura (Solé, 2001); ya establecido como un proceso global que ayuda a la comprensión lectora.

Cuando los lectores tienen un pensamiento analógico, es más fácil para ellos obtener inferencias correctas. El uso de analogías fomenta este tipo de pensamiento fundamental (el pensamiento analógico) en la construcción de conocimientos nuevos y en la comprensión, ya que todo nuevo conocimiento incluirá una búsqueda de aspectos similares entre lo que ya se conoce y lo nuevo, lo familiar y lo no familiar.

El uso de analogías juega un papel muy importante en la reestructuración del marco conceptual de los alumnos principiantes, puede facilitar la comprensión y visualización de conceptos abstractos, que para el desarrollo cognitivo o maduración cognitiva de los niños y niñas de 9 años son difíciles de entender y, además, fomenta el interés por un tema nuevo. Evita los tecnicismos.

Las analogías son estrategias cognitivas, ya que permiten la adquisición y retención del conocimiento, a través de la búsqueda de aspectos similares entre un campo de conocimiento conocido y otro similar, pero menos conocido. Son una ayuda para la memoria, pues posibilitan el acceso y la recuperación de la información previamente almacenada.

La función más importante de la estrategia consiste en que contextualiza el conocimiento que se va a aprender, puntualizando aspectos como el contexto, el desarrollo cognitivo o maduración cognitiva, y los conocimientos previos. Los aspectos mencionados: contexto, desarrollo cognitivo o maduración cognitiva y la información no visual son aspectos que se deben considerar como premisas básicas para la comprensión.

Las analogías contextualizan, porque traen o acercan situaciones parecidas o similares a los lectores, cuando quizás dentro del contexto cultural (comunidad) ese conocimiento no tiene relevancia y, por tanto, no es conocido. Es así como se puede imaginar y crear situaciones similares, que ayuden a comprender conceptos y términos desconocidos y que están fuera del marco cultural del sujeto.

El uso de analogías en la lectura de textos, y aplicadas al alumnado de educación básica primaria, debe estar contextualizado, es decir, el alumnado debe conocer las analogías; de lo contrario, se descontextualiza la posible comparación y se deja a un lado esa parte sociocultural subjetiva de cada sujeto.

Relacionar, comparar y proyectar la información son procesos que permiten al estudiante construir su propio conocimiento. Como se ha mencionado antes, el pensamiento analógico se constituye como el acceso a los procesos de aprendizaje y es este tipo de pensamiento necesario en la lectura de comprensión, ya que toda idea almacenada en la memoria del sujeto requiere de la conjunción con otras para que pueda llevarse a cabo una interacción y modificación de los esquemas mentales.

Por todo lo anterior, se manifiesta en este artículo que las analogías se apegan al enfoque constructivista de la educación. Además, se hace énfasis en el desarrollo del pensamiento analógico como herramienta fundamental para el razonamiento y para el aprendizaje permanente y significativo. 


\section{Referencias bibliográficas}

Ausubel, D. (2002). Adquisición y retención del conocimiento. Una perspectiva cognitiva. Barcelona: Editorial Paidos.

Chadwick, C. (2001). La psicología del aprendizaje del enfoque constructivista. Revista Latinoamericana de Estudios Educativos, XXXI(004), 111-126.

González, A. (2004). Estrategias de comprensión lectora. Madrid, España: Editorial Síntesis.

Gutiérrez, V. (2005). La lectura: una capacidad imprescindible de los ciudadanos del siglo XXI. El caso de México. Anales de documentación, número 008, 91-99.

Instituto Nacional para la Evaluación de la Educación en México [INEE]. (2006). La calidad de la educación básica en México. (Informe anual 2006). México, D. F: Author.

Instituto Nacional para la Evaluación de la Educación en México [INEE]. (2007). Programa Internacional para la Evaluación de los Estudiantes [PISA, 2006 ] en México. México, D. F: Author.

Krippendorff, K. (1990). Metodología del análisis de contenido. Barcelona: Paidós.

Piaget, J. (1969). Psicología y pedagogía. Barcelona: Editorial Ariel.

Sarmiento, C. (1995). Leer y comprender. México: Editorial Planeta.

Smith, F. (1989). Comprensión de la lectura: análisis psicolingüístico de la lectura y su aprendizaje (2a ed.). México: Editorial Trillas.

Solé, I. (2001). Estrategias de lectura. México: Editorial Trillas

Vigotsky, L. (1997). Pensamiento y lenguaje. Teoría del desarrollo cultural de las funciones psíquicas ( $2^{\mathrm{a}}$ ed.). México: Ediciones quinto sol. 